\title{
Multifunctional Switchable Nanocoated Membranes for Efficient Integrated Purification of Oil/Water Emulsions
}

Kai Wang,,${ }^{\text {a b }}$ Huaqiang He, ${ }^{\mathrm{c}}$ Baibing Wei, ${ }^{\text {a }}$ Tian C. Zhang, ${ }^{\mathrm{d}}$ Haiqing Chang, ${ }^{\text {a }}$ Yingqi Li, ${ }^{\text {a Xiaobao }}$ Tian, ${ }^{\text {a }}$ Yubo Fan, ${ }^{\mathrm{b}}$ Ying Liang,,${ }^{\mathrm{a}}$ * Shaojun Yuan ${ }^{\mathrm{c}, ~ *}$

${ }^{a}$ MOE Key Laboratory of Deep Earth Science and Engineering, College of Architecture and

Environmental Engineering, Sichuan University, Chengdu, 610065, P. R. China.

b Beijing Advanced Innovation Center for Biomedical Engineering, Key Laboratory for Biomechanics and Mechanobiology of Ministry of Education, School of Biological Science and Medical Engineering, Beihang University, 100191 Beijing, China.

${ }^{c}$ Low-carbon Technology \& Chemical Reaction Engineering Lab, College of Chemical Engineering, Sichuan University, Chengdu 610065, China.

${ }^{\mathrm{d}}$ Civil \& Environmental Engineering Department, University of Nebraska-Lincoln, Omaha, NE 681820178, USA.

* Corresponding Author e-mail: liangying@scu.edu.cn (Liang Y.); ysj@scu.edu.cn (Yuan S.). 


\section{SUPPLEMENTARY METHODS}

Characterizations. The element distribution of the membrane surface was characterized via an X-ray energy-dispersive spectrometry (EDS, Oxford). Fourier transform infrared spectroscopy (FTIR, Spectrum Two Li10014, PerkinElmer, USA) and X-ray diffraction (XRD, X'pert Pro MPD, PANalytical B.V., Holland) analyses were used to analyzed chemical compositions of the membranes. The pore sizes of the membranes were evaluated by a capillary flow porometer(Porolux 1000, Porometer, Germany). The diffuse reflectance spectra (DRS) analysis was conducted on a UV-vis spectrophotometer (Lambda 750S, PerkinELmer, USA). A noncontact three-dimensional optical profilometer (Veeo Contour GT-K1, Bruker, Germany) was used to measure the surface roughness of the membranes. The roughness factor was obtained by using an atomic force microscope (AFM, NT-MDT, NTEGRA, Russia).

COD measurement. The average chemical oxygen demand contents before and after separation of emulsions were measured according to GB 11914-89 (China). A 2.5-mL aliquot of each testing sample was taken in COD vials. Firstly, $1.5 \mathrm{~mL}$ of $0.125 \mathrm{~mol} \mathrm{~L}^{-1} \mathrm{~K}_{2} \mathrm{Cr}_{2} \mathrm{O}_{7}, 3.5 \mathrm{~mL}$ of $\mathrm{H}_{2} \mathrm{SO}_{4}$, and a pinch of $\mathrm{HgSO}_{4}$ and $\mathrm{Ag}_{2} \mathrm{SO}_{4}$ were added. The vials were tightly sealed and digested at $150^{\circ} \mathrm{C}$ for $2 \mathrm{~h}$ and then were cooled to room temperature. The above mixture was transferred into the flask and 2-3 drops of ferroin indicator were added, followed by being titrated against freshly prepared $0.1 \mathrm{~mol} \mathrm{~L}^{-1}$ $\left(\mathrm{NH}_{4}\right)_{2} \mathrm{Fe}\left(\mathrm{SO}_{4}\right)_{2}$ solution. The titration end point was determined by the appearance of reddish-brown color. For comparison, deionized water (blank) was also run simultaneously. COD was calculated using the following formula:

$$
\mathrm{COD}=\frac{\left(V_{1}-V_{2}\right) \times m \times 8 \times 1000}{V_{3}}
$$

Where $V_{1}$ is volume of titrant used for the sample; $V_{2}$ is volume of the titrant used forblank deionized water; $m$ is the molar concentration of ferrous ammonium sulfate; and $V_{3}$ denotes volume of the sample taken.

Friction test. The abrasion experiment was conducted in which the membrane was placed between a 600 -mesh sandpaperand a $100 \mathrm{~g}$ weight and then pulled forward to $10 \mathrm{~cm}$ as one cycle to assess its mechanical stability.

Measurement of specific surface area. The specific surface area of the composite membrane was detected using an automatic nitrogen adsorption surface meter (Beishide Instrument Technology Co.,3H-2000PM2, Beijing, China) at $77 \mathrm{~K}$.

Determination of the leaching concentration for $\mathbf{Z n}^{2+}$. An inductively coupled plasma optical emission spectroscopy (ICP-OES, Agilent 5110, Agilent Technologies Inc., USA) was used to determine the leaching concentration of zinc ions. 


\section{SUPPLEMENTARY FIGURES}
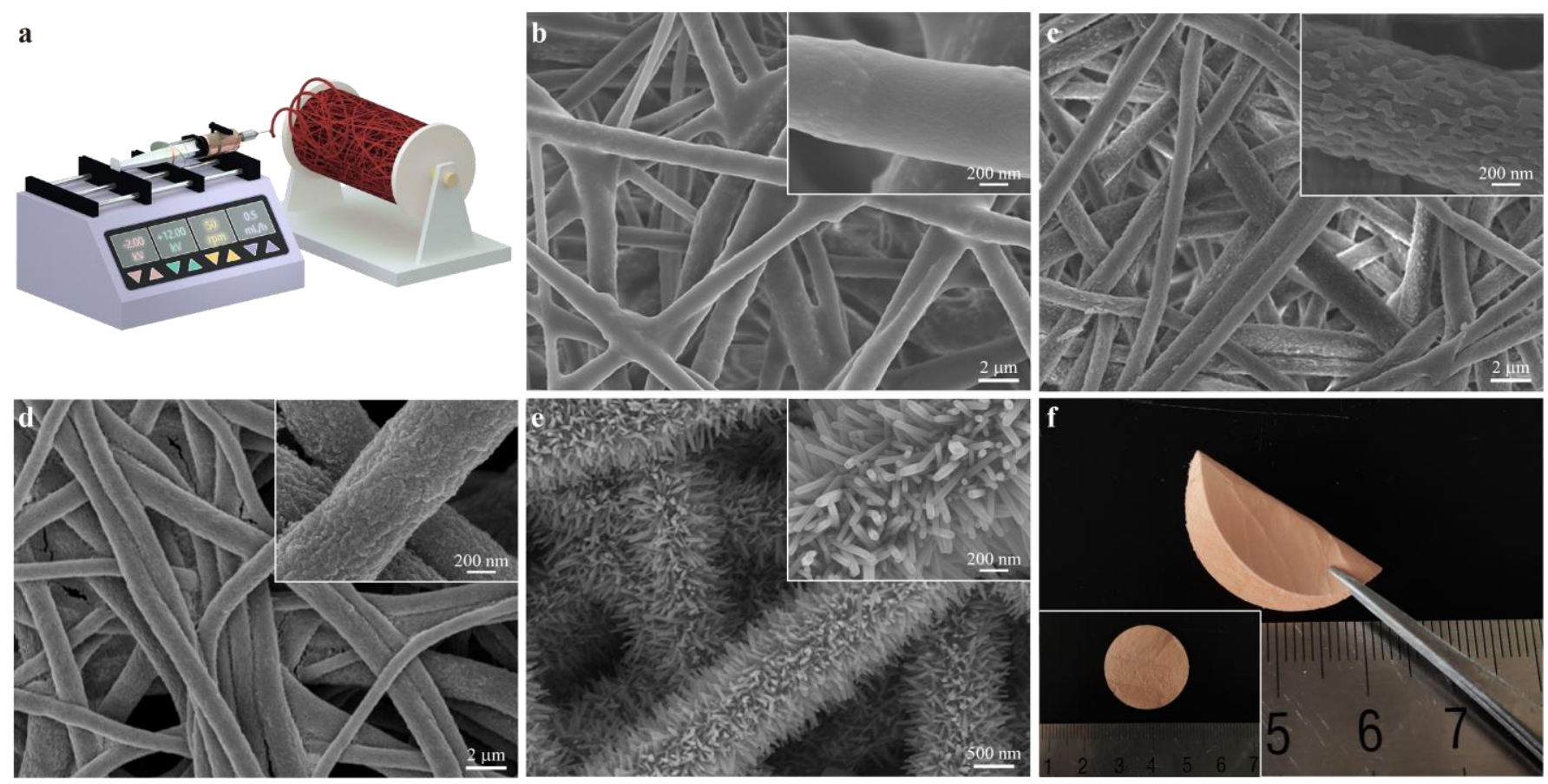

Figure S1. (a) Schematic illustrating the preparation process of electrrospun PVP/PVDF membrane. (b-e) SEM images of the PVP/PVDF, PPVDF, ZnO seeded PPVDF and ZnO-PPVDF membrane, respectively. (f) Photographs showing the flexibility and processibility ofZIF-8@ZnO-PPVDF membrane.

The $\mathrm{ZnO}$ seeds (Figure S1d) were obtained via two in-situ reactions: (i) a neutralization reaction of $\mathrm{Zn}\left(\mathrm{CH}_{3} \mathrm{COO}\right)_{2}+$ $2 \mathrm{NaOH} \rightarrow \mathrm{Zn}(\mathrm{OH})_{2} \downarrow+2 \mathrm{NaCH}_{3} \mathrm{COO}$ at $25^{\circ} \mathrm{C}$; and (ii) a pyrolysis reaction of $\mathrm{Zn}(\mathrm{OH})_{2} \downarrow \rightarrow \mathrm{ZnO} \downarrow+\mathrm{H}_{2} \mathrm{O} \uparrow$ at $125^{\circ} \mathrm{C}$. The crystal seeds were fastened on PPVDF membrane through the adhesion effect of the softened PPVDF surface at $125^{\circ} \mathrm{C}$. The treatment temperature of $125^{\circ} \mathrm{C}$ is in the thermal deformation temperature range of the PVDF materials and leads to the liquation of unstable ending-structures. Mean while, the liquated PPVDF membrane surface can fasten the ZnO seeds firmly, which is of critical importance forgrowing a stable $\mathrm{ZnO}$ nanorods (Figure $\mathrm{S} 1 \mathrm{e}$ ) on the membrane. ${ }^{1}$ 

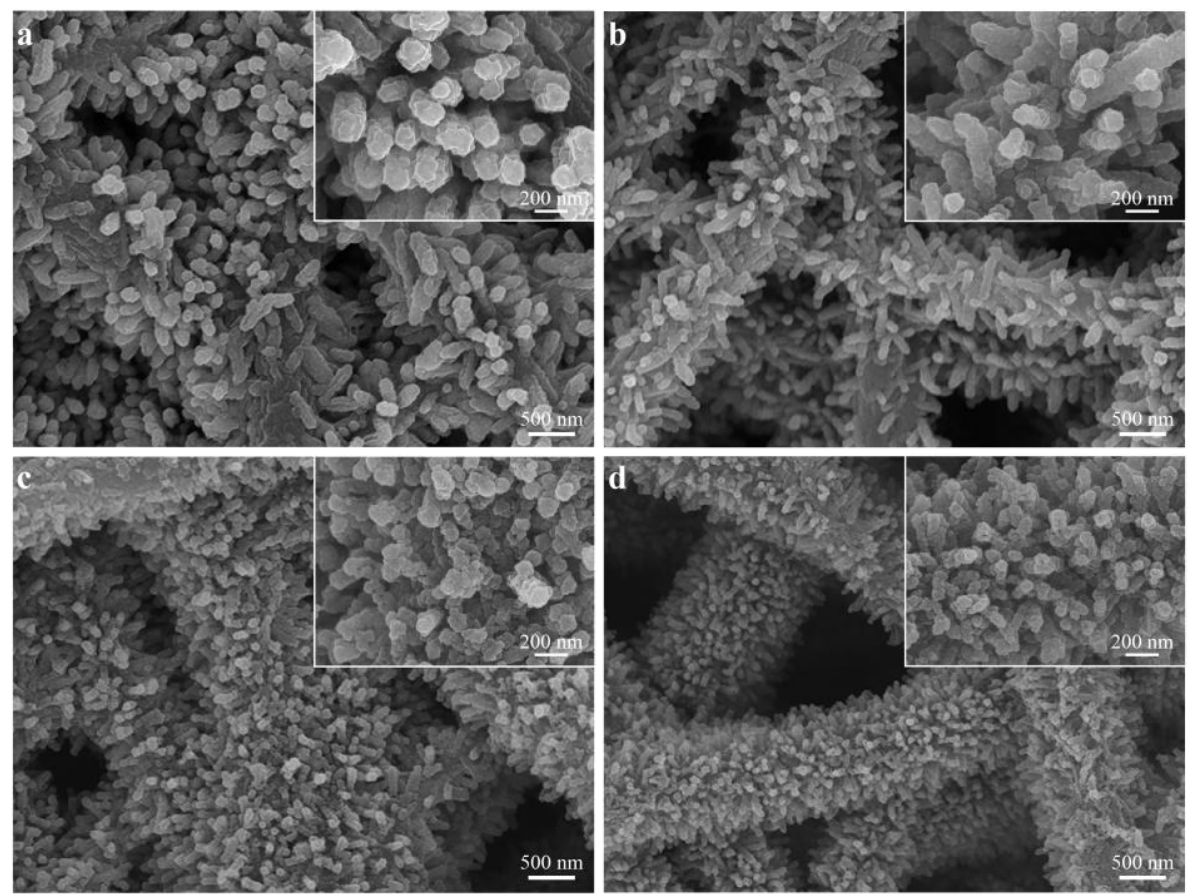

Figure S2. SEM images of the hybrid ZIF-8@ZnO nanorods decorated PPVDF membranes with different reaction Hmim concentrations: (a) 0.4, (b) 0.8 , (c) 1.2 and (d) $2.0 \mathrm{~mol} \mathrm{~L}^{-1}$.

$\mathbf{a}$

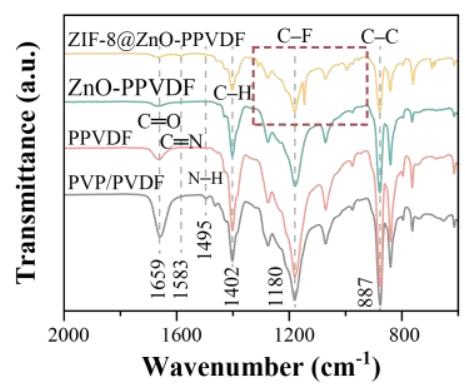

d

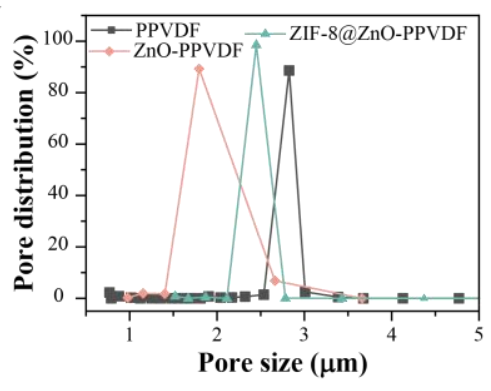

b

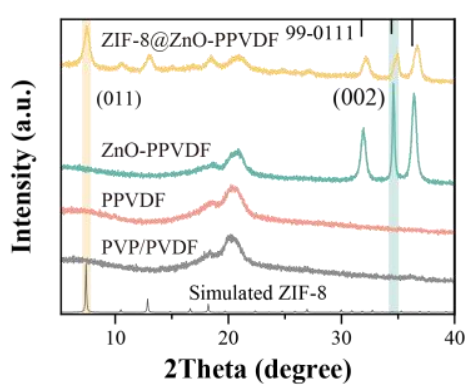

e

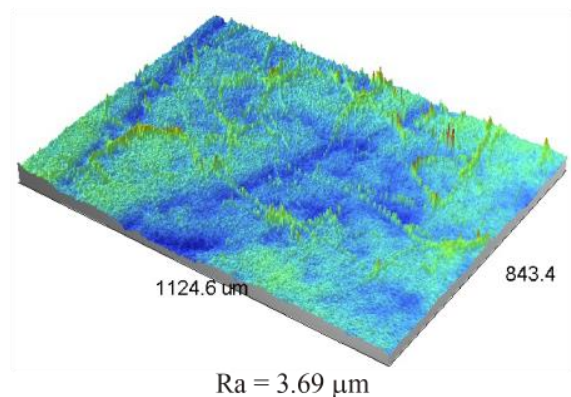

c
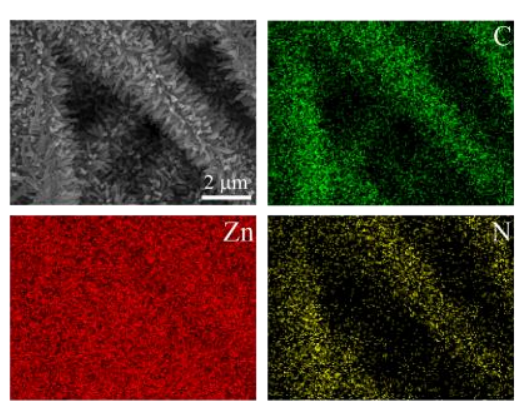

f

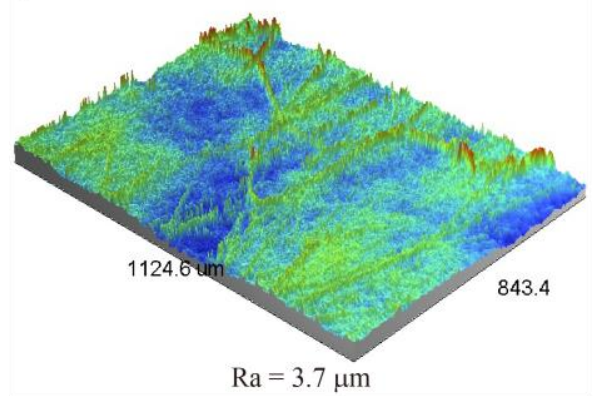

Figure S3. (a) FTIR spectra, (b) XRD patterns, (c) EDS images and (d) pore size distributions of various membranes.

Three-dimensional optical profilometry images of(e) ZnO-PPVDF and (f) ZIF-8@ZnO-PPVDF membranes.

Figure S3a shows that the FTIR spectra of all samples show absorption peaks at 1402 (stretching vibration of C-H), 
1180 (symmetrical stretching of C-F) and $887 \mathrm{~cm}^{-1}$ (skeletal vibration of C-C). ${ }^{2}$ The peaks located at $1659,1583,1495$ $\mathrm{cm}^{-1}$ are assigned to the stretching vibration of $-\mathrm{O}, \mathrm{C}-\mathrm{N}$ and symmetric bending $\mathrm{N}-\mathrm{H}$ primary amines. ${ }^{3}$ The adsorption peak at $1583 \mathrm{~cm}^{-1}(\mathrm{C}-\mathrm{N})$ and red box with dashed lines (ring stretching area) in Figure S3a indicated the successful introduction ofHmim. As shown in Figure S3b, an $\alpha$ phase with a typical peak of $18.4^{\circ}$ and a $\beta$ phase with a peak of $20.8^{\circ}$ of PVDF were observed.

In practice, satisfactory mechanical properties are crucial for the separation materials to maintain its various performance. Thus, a tensile test was conducted to evaluate the mechanical strength of the membrane. Here, the PPVDF, ZnO-PPVDF and ZIF-8@ZnO-PPVDF membranes were selected to perform this test. Figure $1 \mathrm{f}$ demonstrates that as a minor force was loaded, the PPVDF NFM with non-connected structure and smooth fibers would deform easily as confirmed by its low tensile strength (3.5 MPa) and low initial modulus (18.7 MPa). While in the tensile process, less strain for ZnO-PPVDF and ZIF-8@ZnO-PPVDF NFMs had been obtained under the same condition, which was obviously verified by the high tensile strengths (6.5 and $8.4 \mathrm{MPa}$ ) and large initial moduli (139.6 and 169.8 MPa)(Figure 1g). This is mainly because the mechanical properties of the nano-/microfibrous polymer networks are determined by their fibers, interactions of the fibers (e.g., cross-links between fibers), and topologies of the fibrous polymer networks. The networks are entangled or interlocked in a way that they cannot be pulled apart unless the networks are broken. However, the values of elongation at break did not conform to the usual law (decrease as the tensile strength increases). This abnormal phenomenon was because that the interlocked $\mathrm{ZnOnanorods} \mathrm{on} \mathrm{the} \mathrm{coaxial} \mathrm{ZnO}-\mathrm{PPVDF}$ nanofibers would produce effective deformation to enhance the value of elongation at break.

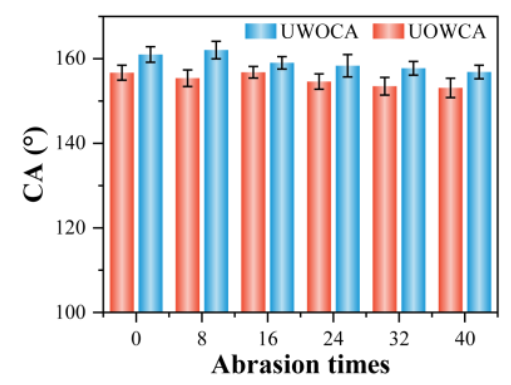

Figure S4. Changes in UWOCAs and UOWCAs of the ZIF-8@ZnO-PPVDF membrane as a function ofabrasion times.

The friction test was conducted in which the membrane was placed between an 800 -grit abrasive paper and a $100 \mathrm{~g}$ weight and then pulled forward to $10 \mathrm{~cm}$ as one cycle to assess its mechanical stability. There was only a minorchange in the wettability of the mesh observed after 40 times abrasion as shown in Figure S3. Such excellent stability could cope 
with the harsh environments and effectively guarantee the long-term use of the as-fabricated membrane.

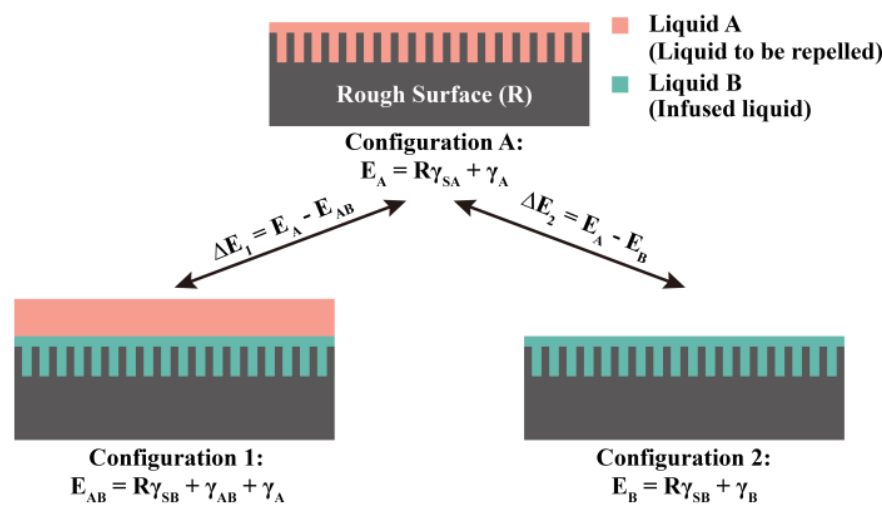

Figure S5. Thermodynamic wetting models. Configurations A and 2 represent thestates that the solid surface is sufficiently infused by liquid A and B, respectively. Configuration 1 refers to the state that solid surface is previously wetted by liquid B with liquid A floating on top ofit.

To determine whether a solid surface would be wetted preferentially by liquid A or liquidB, the total interfacial energies of the wetting configurations $(A, 1,2)$ were analyzed. It is known that the preferred wetting state should possess the lower surface energy. Therefore, to enable the solid surface is preferentially infused by liquid B, we should have the energy states between these wetting configurations that $E_{A}>E_{B}$ or $E_{A B}$, which can be expressed as: ${ }^{4,5}$

$$
\begin{aligned}
& \Delta E_{1}=R\left(\gamma_{\mathrm{SA}}-\gamma_{\mathrm{SB}}\right)-\gamma_{\mathrm{AB}}>0 \\
& \Delta E_{2}=R\left(\gamma_{\mathrm{SA}}-\gamma_{\mathrm{SB}}\right)+\gamma_{\mathrm{B}}-\gamma_{\mathrm{A}}>0
\end{aligned}
$$

Where $\gamma_{\mathrm{SA}}$ and $\gamma_{\mathrm{SB}}$ are the surface tensions of the liquid A-solid interface and liquid B-solid interface, respectively. $\gamma_{\mathrm{A}}$ and $\gamma_{\mathrm{B}}$ represent the surface tensions of the liquid $\mathrm{A}$ (liquid to be repelled) and the liquid $\mathrm{B}$ (infused liquid), respectively. $\gamma_{\mathrm{AB}}$ is the liquid A-liquid $\mathrm{B}$ interface tension, and $R$ is the roughness factor of the solid surface.

Combined with the Young 's equation, equations S2 and S3 can be simplified to: ${ }^{6}$

$$
\begin{aligned}
& \Delta E_{1}=\mathrm{R}\left(\gamma_{B} \cos \theta_{B}-\gamma_{A} \cos \theta_{A}\right)-\gamma_{A B}>0 \\
& \Delta E_{2}=\mathrm{R}\left(\gamma_{B} \cos \theta_{B}-\gamma_{A} \cos \theta_{A}\right)+\gamma_{A}-\gamma_{B}>0
\end{aligned}
$$

where $\theta_{A}$ and $\theta_{B}$ are the intrinsic contact angles of liquid A and liquid B on the smooth surfaces, respectively. When $\Delta E_{1}$ and $\Delta E_{2}$ are all greater than 0 ; liquid B preferentially wets the surface and form a stable liquid-repellent interface. In contrast, when $\Delta E_{1}$ and $\Delta E_{2}$ are all less than 0 , liquid $\mathrm{B}$ will be displaced by liquid $\mathrm{A}$ forming the stable liquid Asolid interface. When $\Delta E_{1}$ and $\Delta E_{2}$ are opposite in sign, liquid B may or may not be displaced by liquid A. 

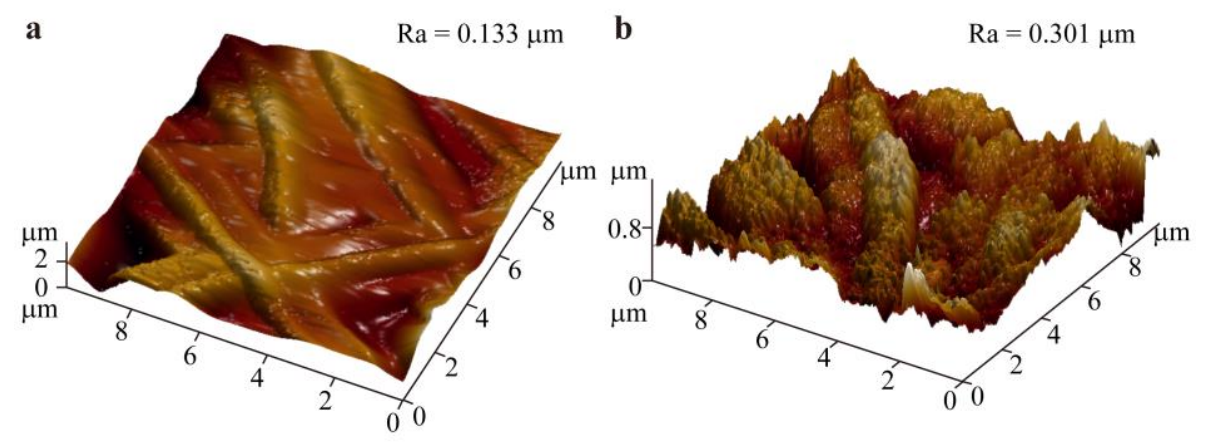

Figure S6. 3D AFM height images of the ZIF-8@ZnO particles (a) and nanorods (b) decorated PPVDF membranes.
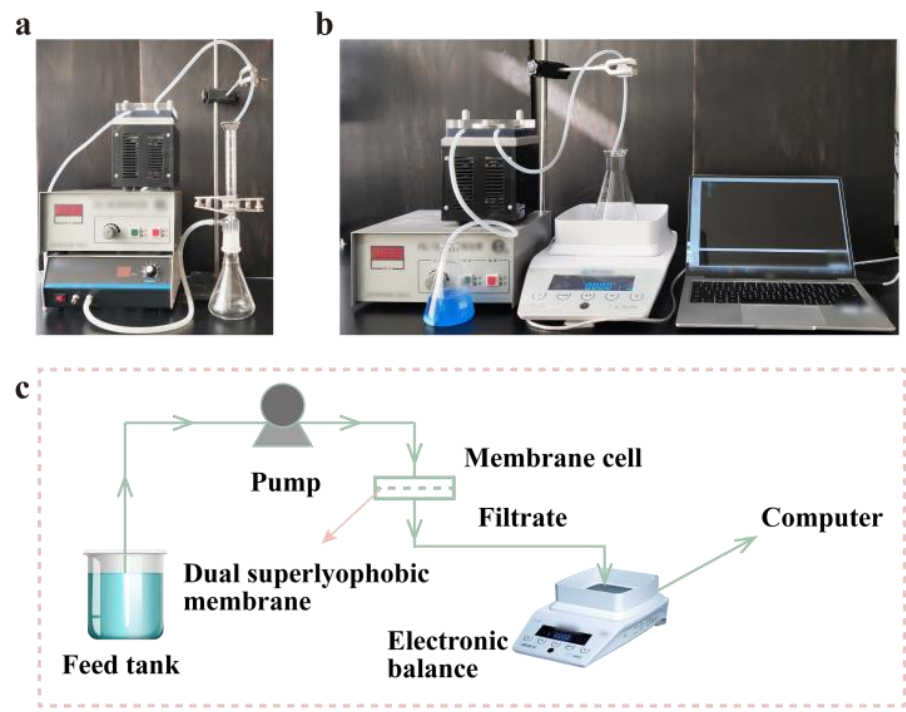

Figure S7. Optical images illustrating the continuous general (a) and complex (b) emulsions separation apparatus. (c) Schematic diagram of the emulsions separation apparatus in (b).

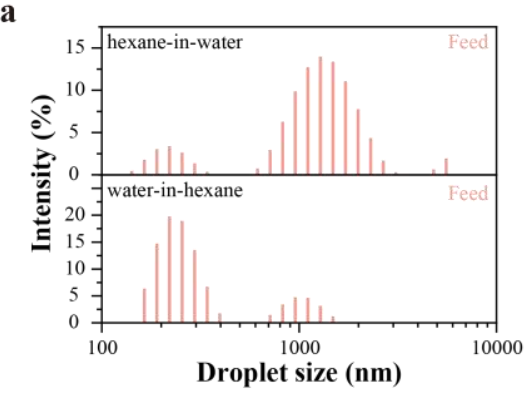

b

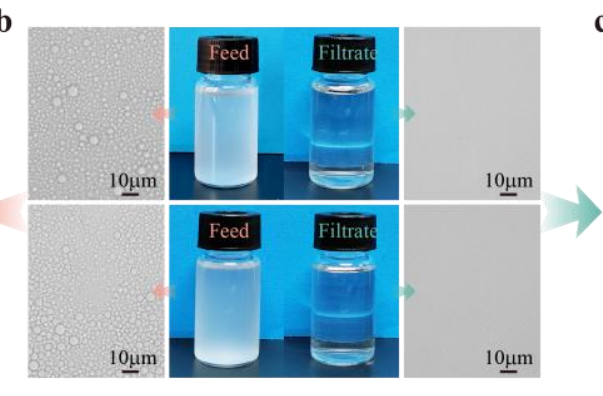

C

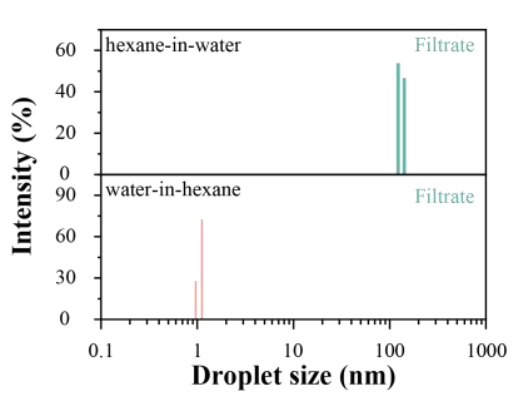

Figure S8. The DLS droplet analysis of n-hexane-in-water SSE (up) and water-in-hexane SSE (down) before (a) and after (c) separation. (b) Optical microscopy images and corresponding digital photos of the corresponding feeds and filtrates 
before and after separation.

a

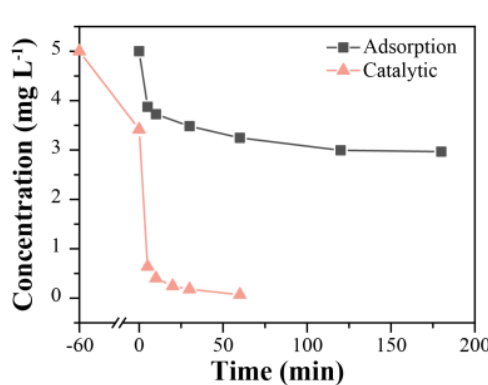

c

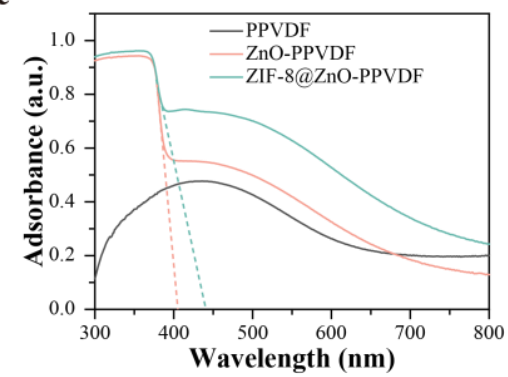

b

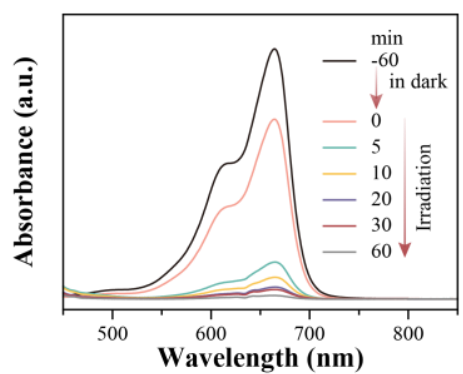

d

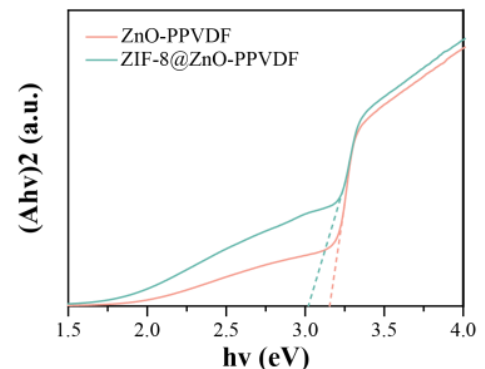

Figure S9. (a) Adsorption and photocatalytic degradation of MB and (b) UV-vis adsorption spectra of MB at different irradiation times through photocatalytic degradation by ZIF-8@ZnO-PPVDF membrane. (c) UV-vis DRS spectra and (d) Tauc plots of the relevant samples.

As shown in Figures S9a \& b, the absorbance at 664 of MB weakened gradually and almost disappeared after 60 min irradiation. The optical property of the ZIF-8@ZnO-PPVDF membrane was further evaluated by the UV-vis diffuse reflectance spectra (DRS) spectra. As shown in Figures S9c \& d, a weak adsorption edgeappeared in the PPVDF membrane adsorption curve, which indicates its poor visible photoactivity. For the ZnO-PPVDF membrane, an intense absorbance peak located around 300-380 $\mathrm{nm}$ and a little increase of the light absorption over the whole visible light region could be observed, which can be attributed to the strong UV absorption properties of $\mathrm{ZnO},{ }^{7}$ the formation of conjugated structures and the color of PPVDF membrane changed from white into brownish red. With the formation of hybrid ZIF-8@ZnO nanorods, an obvious increase of the light absorption over the whole light region could be observed, which can be attributed to the formation of conjugated structures. In addition, the band-edge emission peak located at the visible-light range ( $\lambda \sim$ $440 \mathrm{~nm}, 2.82 \mathrm{eV}$ ). The absorbance coefficient of the ZIF-8@ZnO-PPVDF NFM was calculated using Tauc plot. The extrapolation of the linear region revealed the absorption bands located at $3.02 \mathrm{eV}$. As the theoretical bandgaps of the $\mathrm{ZnO}$ and ZIF-8 are 3.2 and $3.87 \mathrm{eV}$, the red shift of the bandgap might be due to the dopant or heterojunction among the $\mathrm{ZnO}$, 
ZIF-8 crystalline and PVDF polymer. The photoelectrons and vacancies generated by the visible light irradiation can react with water and oxygen to generate high activity superoxide anions and hydroxyl radicals, which can further decompose organic contaminants adsorbed inside the separation membrane.

a

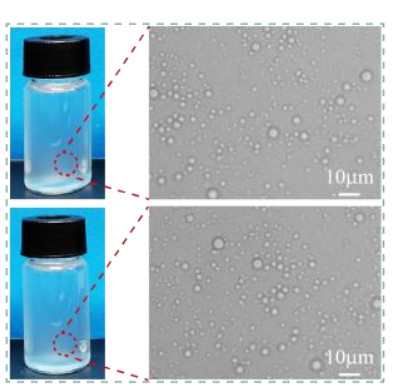

d

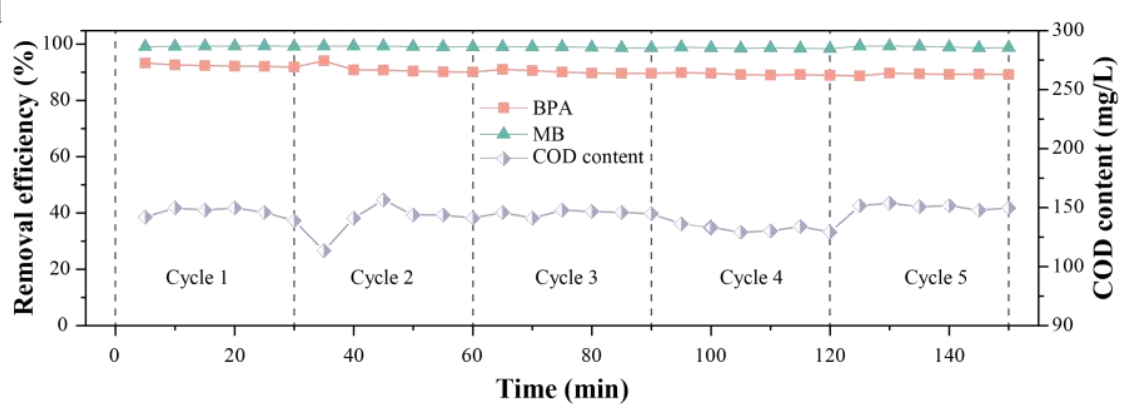

c
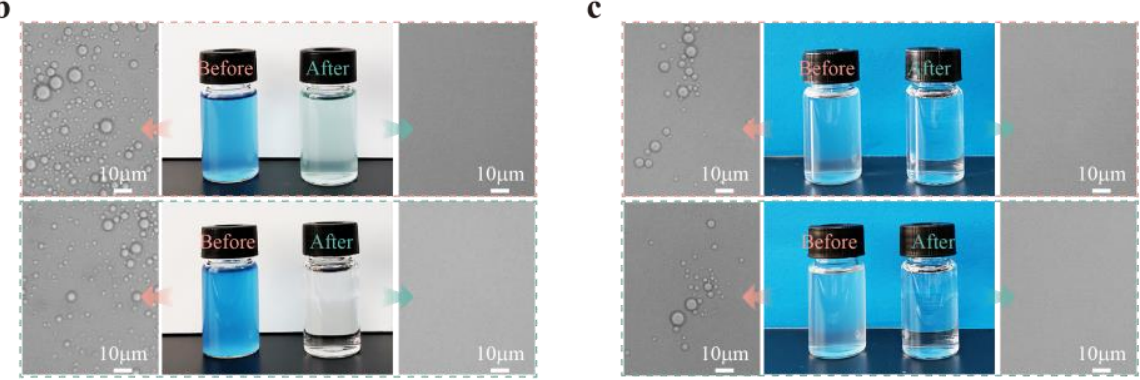

e

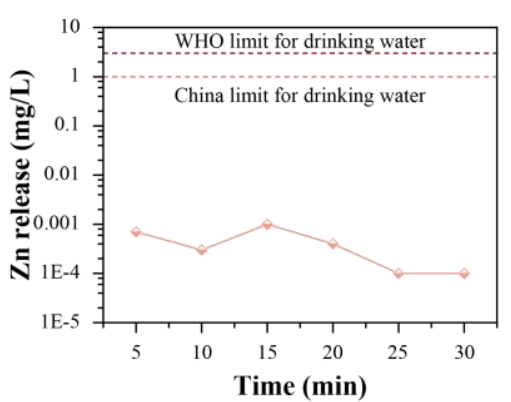

Figure S1 0. (a) The digital photos of petroleum ether-in-water emulsion after 80 min stirring mixed with nothing (top) with light irradiation and ZIF-8@ZnO-PPVDF membrane (bottom) without (bottom) light irradiation. Treatment results of petroleum ether-in-water SSE: Optical microscopy images and corresponding digital photos of emulsions with MB (b) and BPA (c) in water before and after 80 min visible light irradiation (top) and filtration (bottom); (d) The COD contents, MB, and BPA removal efficiencies of the filtrates for emulsion with MB/BPAin water over five cycles. (e) The leaching of Zn ions during one treatment cycle.
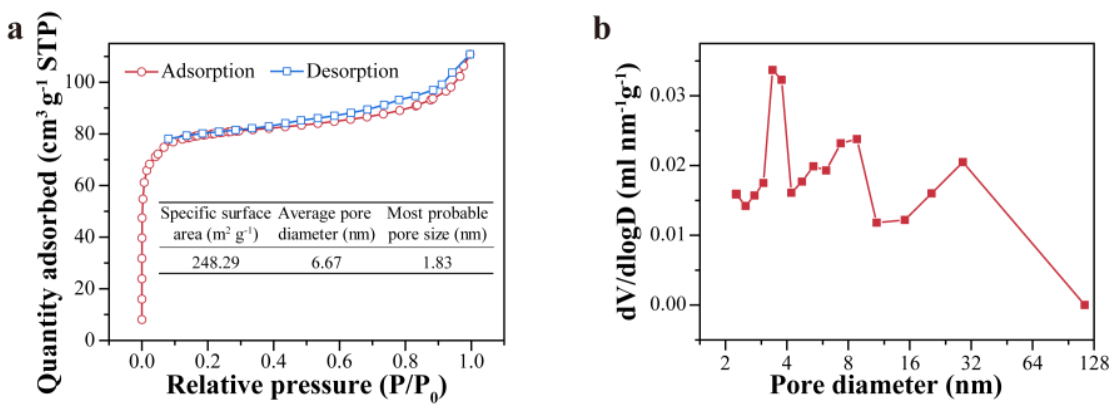
Figure S11. (a) $\mathrm{N}_{2}$ adsorption-desorption isotherm of ZIF-8@ZnO-PPVDF membrane and (b) corresponding pore distribution analysis using the BJH method.

PVP removal by washing and the growth of hybrid ZIF-8@ZnOnanorods (to convert PVP/PVDF to ZIF-8@ZnO-PPVDF) significantly increased the BET surface area of the membrane (i.e., from 1.85 to $248.29 \mathrm{~m}^{2} \mathrm{~g}^{-1}$, Figure S11). ${ }^{7}$ The value was much higher than previously reported electrospun fiber membranes (12.5 to $\left.117.2 \mathrm{~m}^{2} \mathrm{~g}^{-1}\right)$ likely due to pore generation. ${ }^{8}$

a

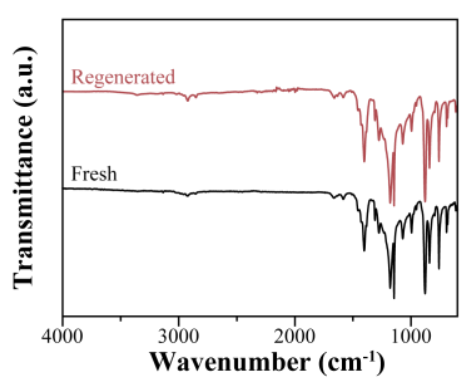

b

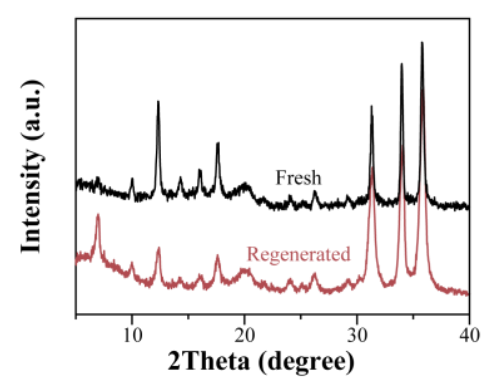

Figure S12. (a) FTIR spectrum and (b) XRD pattern of fresh and regenerated (after 5 cycles) ZIF-8@ZnO-PPVDF membrane. 


\section{SUPPLEMENTARY TABLES}

Table S1. Comparison of the governing relationships with experimental results in a hexane-water-solid system.

\begin{tabular}{|c|c|c|c|c|c|c|c|c|c|c|c|}
\hline \multirow{2}{*}{ Liquid A } & \multirow{2}{*}{ Liquid B } & \multirow{2}{*}{$\mathrm{R}$} & $\gamma_{A}$ & $\gamma_{B}$ & $\gamma_{A B}$ & $\theta_{A}$ & $\theta_{B}$ & $\Delta E_{1}$ & $\Delta E_{2}$ & \multicolumn{2}{|c|}{ Stale film? } \\
\hline & & & \multicolumn{3}{|c|}{$\mathrm{mJ} \mathrm{m}^{-2}$} & \multicolumn{2}{|c|}{$\circ$} & \multicolumn{2}{|c|}{$\mathrm{mJ} \mathrm{m}^{-2}$} & Theory & Exp \\
\hline water & hexane & 1.69 & 71.3 & 18.4 & 49.3 & 57.3 & $\sim 0$ & -83.3 & 18.9 & $\mathrm{Y} / \mathrm{N}$ & DL \\
\hline hexane & water & 1.69 & 18.4 & 71.3 & 49.3 & $\sim 0$ & 57.3 & -15.3 & -18.9 & $\mathrm{~N}$ & DL \\
\hline
\end{tabular}

Note: "Y" indicates that liquid B can form a stable liquid film on the surface and is not replaced by liquid A. "N" indicates that the liquid A-solid interface is more stable, and liquid B will be displaced by liquid A. "DL" represents the under-liquid dual superlyophobic surfaces. $\theta_{A}$ and $\theta_{B}$ represent intrinsic contact angles, which are tested on the ZIF-8@ZnO particles decorated PPVDF membranes.

Table S2. Under-water oil contact angles (UWOCAs) and under-oil water contact angles (UOWCAs) of the hybrid ZIF8@ZnO nanorods decorated PPVDF membranes obtained with different Hmim concentrations.

\begin{tabular}{ccc}
\hline Concentration of $\operatorname{Hmim}(\mathrm{M})$ & UWOCAs $\left(^{\circ}\right)$ & UOWCAs $\left(^{\circ}\right)$ \\
\hline 0.4 & 155 & 156 \\
0.8 & 157 & 158 \\
1.2 & 161 & 157 \\
1.6 & 159 & 154 \\
2.0 & 157 & 156 \\
\hline
\end{tabular}

Table S3. Average, minimum, and maximum pore diameters of various membranes.

\begin{tabular}{cccc}
\hline & $\begin{array}{c}\text { Average pore diameter } \\
(\mu \mathrm{m})\end{array}$ & $\begin{array}{c}\text { Minimum pore diameter } \\
(\mu \mathrm{m})\end{array}$ & $\begin{array}{c}\text { Maximum pore } \\
\text { diameter }(\mu \mathrm{m})\end{array}$ \\
\hline PPVDF & 2.92 & 2.67 & 3.39 \\
ZnO-PPVDF & 2.23 & 1.19 & 2.66 \\
ZIF-8@ZnO-PPVDF & 2.62 & 1.96 & 2.78 \\
\hline
\end{tabular}




\section{SUPPLEMENTARY MOVIES}

Movie S1: Emulsion separation process under the driving of 0.1 bar pressure by vacuum pump utilizing the petroleum ether-in-water emulsion as an example.

Movie S2: Emulsion separation process under the driving of 0.1 bar pressure by vacuum pump utilizing the water-inpetroleum etheremulsion as an example. 


\section{REFERENCES}

(1) Zhang, M.; Wang, L.; Feng, S.; Zheng, Y. A Strategy of Antifogging: Air-Trapped Hollow Microsphere Na nocomposites. Chem. Mater. 2017, 29, 2899-2905.

(2) An, A. K.; Guo, J.; Lee, E.; Jeong, S.; Zhao, Y.; Wang, Z.; Leiknes, T. PDMS/PVDF Hybrid Electrospun Membrane with Superhydrophobic Property and Drop Impact Dynamics for Dyeing Wastewater Treatment Using Membrane Distillation. J. Membrane Sci. 2017, 525, 57-67.

(3) Zhang, Y.; Guan, J.; Wang, X.; Yu, J.; Ding, B. Balsam-Pear-Skin-Like Porous Polyacrylonitrile Nanofibrous Membranes Grafted with Polyethyleneimine for Postcombustion $\mathrm{CO}_{2}$ Capture. ACS Appl. Mater. Interfaces 2017,9, 41087-41098.

(4) Wang, Y.; Di, J.; Wang, L.; Li, X.; Wang, N.; Wang, B.; Tian, Y.; Jiang, L.; Yu, J. Infused-Liquid-Switchable Porous Na nofibrous Membranes for Multiphase Liquid Separation. Nat. Commun. 2017, 8, 575.

(5) Wong, T.; Kang, S. H.; Tang, S. K. Y.; Smythe, E. J.; Hatton, B. D.; Grinthal, A.; Aizenberg, J. Bioinspired SelfRepairing Slippery Surfaces with Pressure-Stable Omniphobicity. Nature 2011,477,443-447.

(6) Wang, Q.; Wang, Y.; Wang, B.; Liang, Z.; Di J; Yu, J. Under-Liquid Dual Superlyophobic Nanofibrous Polymer Membranes Achieved by Coating Thin-Film Composites: A Design Principle. Chem. Sci. 2019, 10, 6382-6389.

(7) Wang, K.; Zhang, T. C.; Wei, B.; Chen, S.; Liang, Y.; Yuan, S. Durable CNTs Reinforced Porous Electrospun Superhydrophobic Membrane for Efficient Gravity Driven Oil/Water Separation. Colloid. Surface. A 2021, 608, 125342.

(8) Lee, C.; Javed, H.; Zhang, D.; Kim, J.; Westerhoff, P.; Li, Q.; Alvarez, P. J. J. Porous Electrospun Fibers Embedding $\mathrm{TiO}_{2}$ for Adsorption and Photocataly tic Degradation of Wa ter Pollutants. Environ. Sci. Technol. 2018, 52, 4285-4293. 